\section{Test, multipler diagnostischer}

R.-D. Hilgers ${ }^{1}$, N. Heussen ${ }^{1}$ und S. Stanzel ${ }^{2}$

${ }^{1}$ Institut für Medizinische Statistik, Universitätsklinikum der RWTH Aachen, Aachen, Deutschland

${ }^{2}$ DKFZ Heidelberg, Heidelberg, Deutschland

Englischer Begriff multiple diagnostic testing

Definition Man spricht von einem multiplen diagnostischen Test, wenn mehrere unterschiedliche diagnostische Tests $(\triangleright$ Test, diagnostischer) nacheinander oder gleichzeitig durchgeführt werden, um eine gemeinsame diagnostische Aussage zu treffen.

Beschreibung Bei der mehrfachen Anwendung diagnostischer Tests kann vereinfachend zunächst zwischen parallelen
( $\triangleright$ Test, paralleler diagnostischer) und seriellen Tests ( $\triangleright$ Test, serieller diagnostischer) unterschieden werden. In der Praxis liegen häufig Mischformen dieser Teststrategien vor. Der Formulierung einer Entscheidungsregel, die die Grundlage für die Diagnose auf der Basis aller Einzeltestergebnisse bildet, kommt eine besondere Bedeutung zu. Dabei sind sowohl unterschiedliche Gewichtungen, etwa hinsichtlich der Sicherheit, Kosten oder Falschdiagnosen, als auch unterschiedliche Reihenfolgen denkbar. Eine detaillierte Analyse der einzelnen Tests, insbesondere deren Konsequenzen bildet die Grundlage für einen „diagnostischen“ Entscheidungsbaum.

\section{Literatur}

Hilgers R-D, Bauer P, Scheiber V (2002) Einführung in die Medizinische Statistik. Springer, Berlin/Heidelberg/New York 